\title{
Uncertainty contribution of a wind turbine to the electric field of a DVOR antenna as a function of wind direction and rotor position
}

\author{
Sergei Sandmann and Heyno Garbe \\ Institute of Electrical Engineering and Measurement Technology, Leibniz Universität Hannover, Hannover, Germany \\ Correspondence to: Sergei Sandmann (sandmann@geml.uni-hannover.de)
}

Received: 24 January 2016 - Accepted: 13 May 2016 - Published: 28 September 2016

\begin{abstract}
The presence of a wind turbine (WT) has the potential to distort electromagnetic fields emitted by terrestrial radio navigation aids. In this paper especially the field distortion of a Doppler Very High Frequency Omnidirectional Radio Range (DVOR) surveillance navigation system is investigated as a function of wind direction and rotor position. Therefor, the field distribution of a DVOR is simulated in the surrounding of a WT for 104 combinations of the angles of wind direction and rotor position. Furthermore, these calculations are executed for two different rotor diameters and 10 steps of distance between DVOR and WT in the range of $10 \mathrm{~km}$. Based on the calculated data a method to estimate the maximum field distortion is developed. It is shown that the presented method allows to approximate the worst case field distortion with the results of two general simulation setups. Eliminating the need of simulating all possible geometric constellations of the WT this method hereby offers the benefit of significantly reduced simulation effort.
\end{abstract}

\section{Introduction}

Caused by the facilitation of using renewable energy sources the ever growing number of wind turbines (WT) leads to a rising probability of disturbing interaction with radio surveillance navigation systems (Gallardo-Hernando et al., 2011; Van Lil et al., 2009). To guarantee their reliability the installation of WTs is prohibited in specified nearby area (ICAO EUR DOC 015, 2015). Especially for the Doppler Very High Frequency Omnidirectional Radio Range (DVOR) this approach often leads to overcautious decisions which cause an unnecessary blocked high investment volume (BWE, 2014). A preferable procedure is to predict the disturbing potential of every single WT on the specific DVOR, so that applica- tions for new WT installations are only rejected based on more and better scientific considerations. A typical way to calculate a prediction is performed by simulating the electromagnetic conditions. Since a WT has two degrees of freedom composed of the angles of wind direction and rotor position, one of the difficulties is taking into account the WT's interaction with the surrounding electromagnetic field as a function of the geometrical constellation. A straight way forward is to determine the worst case or the statistical distribution by stepwise simulating the WT in all possible constellations. Depending on the step width this leads to a high number of simulation iterations, whereat one single simulation setup with a WT of common size already requires a high amount of simulation resources due to the very large involved surfaces to consider.

Based on this context, this paper describes a method to approximate the worst case electromagnetic field distortion by a WT. The field used in this investigation is the omnidirectional carrier wave generated by a DVOR with a frequency of $112 \mathrm{MHz}$. After analyzing the field distortions with stepwise simulations of 104 non-redundant WT constellations, according to Figs. 1a and b, regularities are derived from the results and used for methodical approximation of the worst case interaction with the surrounding field. With the introduced method the results of only two general simulation setups are needed for calculating the approximated field values: simulation of field distribution in the original setup without WT at all and with a simplified rotationally symmetric substitution model (RSSM) of the WT, which except for the missing blades is as similar as possible to the original model, as shown in Fig. 2.

As only the carrier wave is investigated in this paper, the results are correspondingly referred to field strengths only 


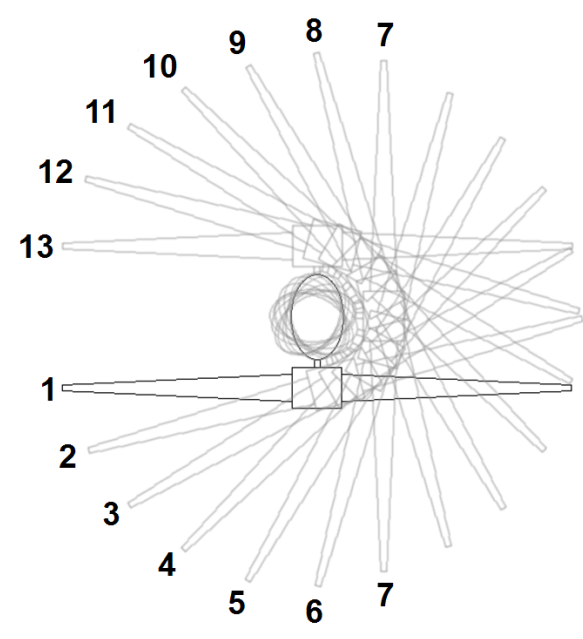

(a)

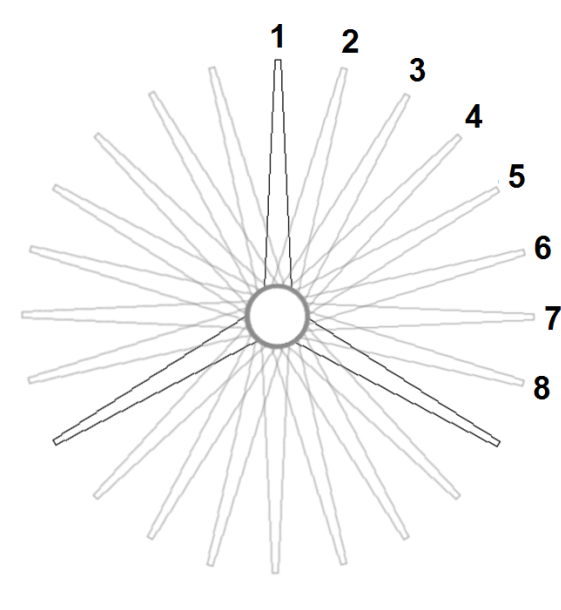

(b)

Figure 1. Illustration of the angles of wind direction (a) and rotor position (b).

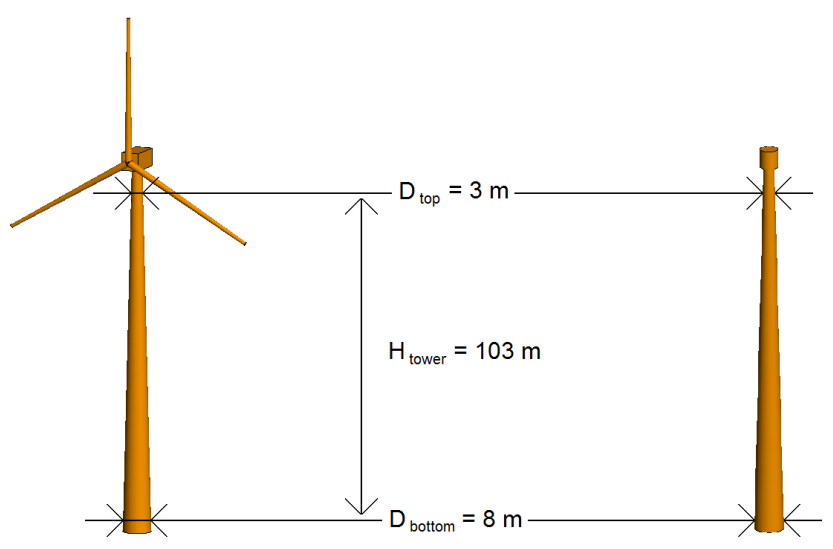

Figure 2. Dimensions of wind turbine (WT) simulation model and simplified rotationally symmetric substitution model (RSSM).

instead of the transmitted angle information used for navigation which is contained in the frequency side bands.

\section{Simulation setup}

As mentioned in the introduction the omnidirectional carrier wave of a DVOR surveillance antenna, consisting of 51 Alford Loop (AL) antennas, is used as the source of the simulation setup. Therefor, according to Sandmann et al. (2015), only the center AL antenna of the DVOR model shown in Fig. 3 is fed, whereas the remaining AL antennas are complex-conjugated impedance matched.

While the DVOR antenna is always placed in the origin, the electromagnetic field strength is calculated in a circular volume around the WT, placed in a variable distance $X$ between 1 and $10 \mathrm{~km}$ on the U-axis, as illustrated in Fig. 4. The unusual placement of the field calculation volume around the WT instead the emitting antenna was chosen expecting the WT's distortion contribution in an approximately constant range along the azimuth, as described more detailed in Sect. 3.3. The calculation points of the field strength in the described volume are arranged in 100 coaxial circles representing orbit flights (OF) with different radii and heights, marked as intersection points in cross section view in Fig. 5.

The overall parametric simulation values for the distance $X$ of the WT on the $\mathrm{U}$-axis, the rotor diameter $D$ and the angle of wind direction $\alpha$ and rotor position $\beta$ according to Fig. 1 , as well as the OFs' distances $R$ and altitudes $H$, are summarized in Table 1.

The described simulation setup was carried out with all possible combinations of these parameters which corresponds to 2080 variations. For all calculations the Multilevel Fast Multipole Method (MLFMM) (van Tonder et al., 2005) of the software tool FEKO (Altair FEKO, 2015) was used. The DVOR and WT models are treated as fully metallic with perfect electrical conductivity.

\section{Simulation results and estimation method}

\subsection{Simulation results}

As a result of the simulation the distribution of the electric field strength along the coaxial OF introduced in Sect. 2 can be indicated as shown in Fig. 6 for an OF with $X=1 \mathrm{~km}$, $D=82 \mathrm{~m}, R=6 \mathrm{NM}$ and $H=6000 \mathrm{ft}$. The red graph indicates the results for one single exemplary constellation of wind direction angle $\alpha$ and rotor angle $\beta$. For all possible constellations the appropriate 104 graphs establish the tube shaped formation shown in green. 


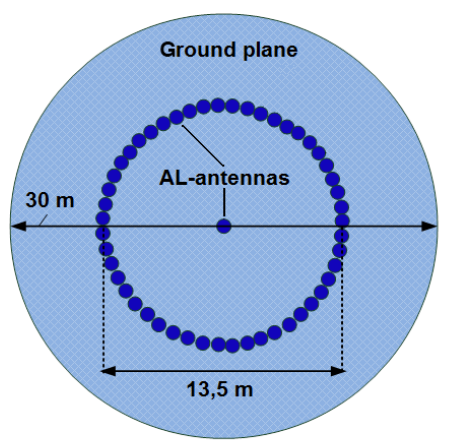

(a)

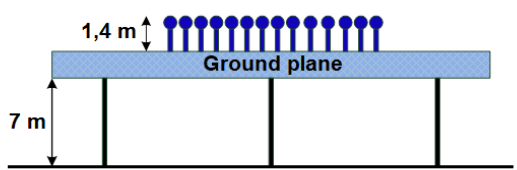

(b)

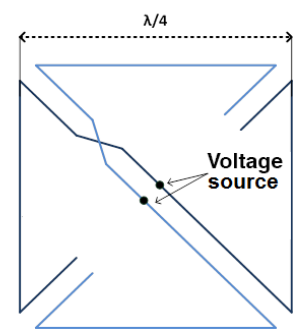

(c)

Figure 3. Simulation model of DVOR antenna consisting of 51 Alford Loop antennas.

Table 1. Summary of all parameter values used for simulations.

\begin{tabular}{|c|c|c|c|c|c|c|c|c|c|c|}
\hline$X$ & $1 \mathrm{~km}$ & $2 \mathrm{~km}$ & $3 \mathrm{~km}$ & $4 \mathrm{~km}$ & $5 \mathrm{~km}$ & $6 \mathrm{~km}$ & $7 \mathrm{~km}$ & $8 \mathrm{~km}$ & $9 \mathrm{~km}$ & $10 \mathrm{~km}$ \\
\hline$D$ & $82 \mathrm{~m}$ & $114 \mathrm{~m}$ & & & & & & & & \\
\hline$\alpha$ & $\begin{array}{l}\text { Pos. } 1 \\
0^{\circ}\end{array}$ & $\begin{array}{c}\text { Pos. } 2 \\
15^{\circ}\end{array}$ & $\begin{array}{l}\text { Pos. } 3 \\
30^{\circ}\end{array}$ & $\begin{array}{c}\text { Pos. } 4 \\
45^{\circ}\end{array}$ & $\begin{array}{l}\text { Pos. } 5 \\
60^{\circ}\end{array}$ & $\begin{array}{c}\text { Pos. } 6 \\
75^{\circ}\end{array}$ & $\begin{array}{c}\text { Pos. } 7 \\
90^{\circ}\end{array}$ & $\begin{array}{c}\text { Pos. } 8 \\
105^{\circ}\end{array}$ & $\begin{array}{c}\text { Pos. } 9 \\
120^{\circ}\end{array}$ & $\begin{array}{c}\text { Pos. } 10 \\
135^{\circ}\end{array}$ \\
\hline$\alpha$ & $\begin{array}{c}\text { Pos. } 11 \\
150^{\circ}\end{array}$ & $\begin{array}{c}\text { Pos. } 12 \\
165^{\circ}\end{array}$ & $\begin{array}{c}\text { Pos. } 13 \\
180^{\circ}\end{array}$ & & & & & & & \\
\hline$\beta$ & $\begin{array}{l}\text { Pos. } 1 \\
0^{\circ}\end{array}$ & $\begin{array}{c}\text { Pos. } 2 \\
15^{\circ}\end{array}$ & $\begin{array}{l}\text { Pos. } 3 \\
30^{\circ}\end{array}$ & $\begin{array}{l}\text { Pos. } 4 \\
45^{\circ}\end{array}$ & $\begin{array}{l}\text { Pos. } 5 \\
60^{\circ}\end{array}$ & $\begin{array}{c}\text { Pos. } 6 \\
75^{\circ}\end{array}$ & $\begin{array}{c}\text { Pos. } 7 \\
90^{\circ}\end{array}$ & $\begin{array}{c}\text { Pos. } 8 \\
105^{\circ}\end{array}$ & & \\
\hline$R$ & $\begin{array}{l}1 \mathrm{NM} \\
\underset{1.8 \mathrm{~km}}{\approx}\end{array}$ & $\begin{array}{l}2 \mathrm{NM} \\
\approx \\
3.7 \mathrm{~km}\end{array}$ & $\begin{array}{l}3 \mathrm{NM} \\
\approx \\
5.6 \mathrm{~km}\end{array}$ & $\begin{array}{c}4 \mathrm{NM} \\
\approx \\
7.4 \mathrm{~km}\end{array}$ & $\begin{array}{c}5 \mathrm{NM} \\
\underset{9.3 \mathrm{~km}}{\approx}\end{array}$ & $\begin{array}{c}6 \mathrm{NM} \\
\approx \\
11.1 \mathrm{~km}\end{array}$ & $\begin{array}{c}7 \mathrm{NM} \\
\approx \\
13.0 \mathrm{~km}\end{array}$ & $\begin{array}{c}8 \mathrm{NM} \\
\approx \\
14.8 \mathrm{~km}\end{array}$ & $\begin{array}{c}9 \mathrm{NM} \\
\approx \\
16.7 \mathrm{~km}\end{array}$ & $\begin{array}{c}10 \mathrm{NM} \\
\approx \\
18.5 \mathrm{~km}\end{array}$ \\
\hline$H$ & $\begin{array}{c}1000 \mathrm{ft} \\
\approx \\
0.3 \mathrm{~km}\end{array}$ & $\begin{array}{c}2000 \mathrm{ft} \\
\approx \\
0.6 \mathrm{~km}\end{array}$ & $\begin{array}{l}3000 \mathrm{ft} \\
\underset{0.9 \mathrm{~km}}{\approx}\end{array}$ & $\begin{array}{l}4000 \mathrm{ft} \\
\approx \\
1.2 \mathrm{~km}\end{array}$ & $\begin{array}{l}5000 \mathrm{ft} \\
\underset{1.5 \mathrm{~km}}{\approx}\end{array}$ & $\begin{array}{l}6000 \mathrm{ft} \\
\underset{1.8 \mathrm{~km}}{\approx}\end{array}$ & $\begin{array}{l}7000 \mathrm{ft} \\
\approx \\
2.1 \mathrm{~km}\end{array}$ & $\begin{array}{l}8000 \mathrm{ft} \\
\approx \\
2.4 \mathrm{~km}\end{array}$ & $\begin{array}{l}9000 \mathrm{ft} \\
\approx \\
2.7 \mathrm{~km}\end{array}$ & $\begin{array}{c}10000 \mathrm{ft} \\
\approx \\
3.0 \mathrm{~km}\end{array}$ \\
\hline
\end{tabular}

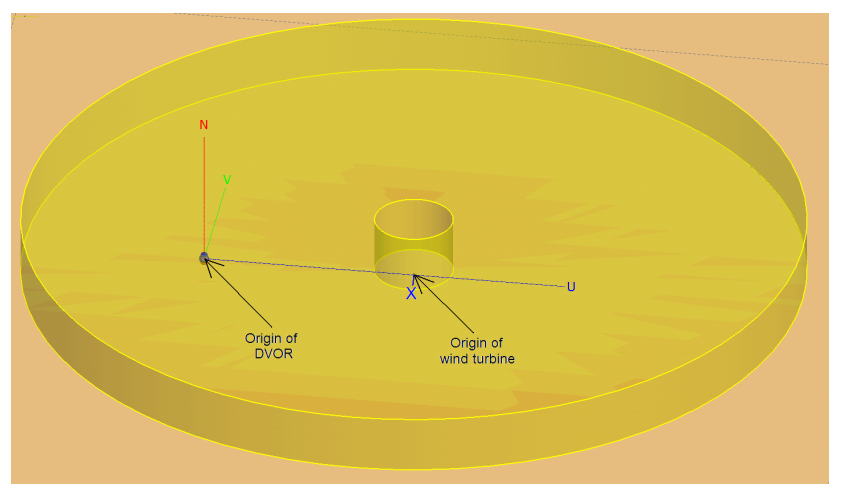

Figure 4. Overview of the simulation setup.

The rising field strength values in the azimuth area of nearly $200^{\circ}$ are caused by the closeness to the DVOR antenna at this region since the beginning of the azimuth $\gamma$ is defined on the averted part of the U-axis, as shown in Fig. 5.

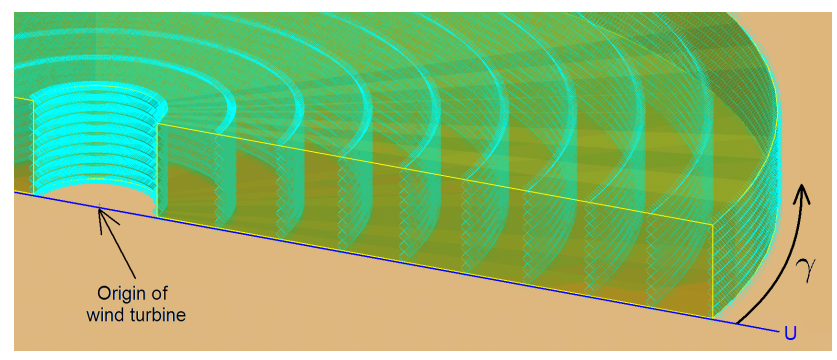

Figure 5. Illustration of the field strength calculation points arranged in 100 coaxial circles representing orbit flights in cross section view.

The deviation from the exact value of $180^{\circ}$ is caused by unbalanced capacitances in the AL model. 


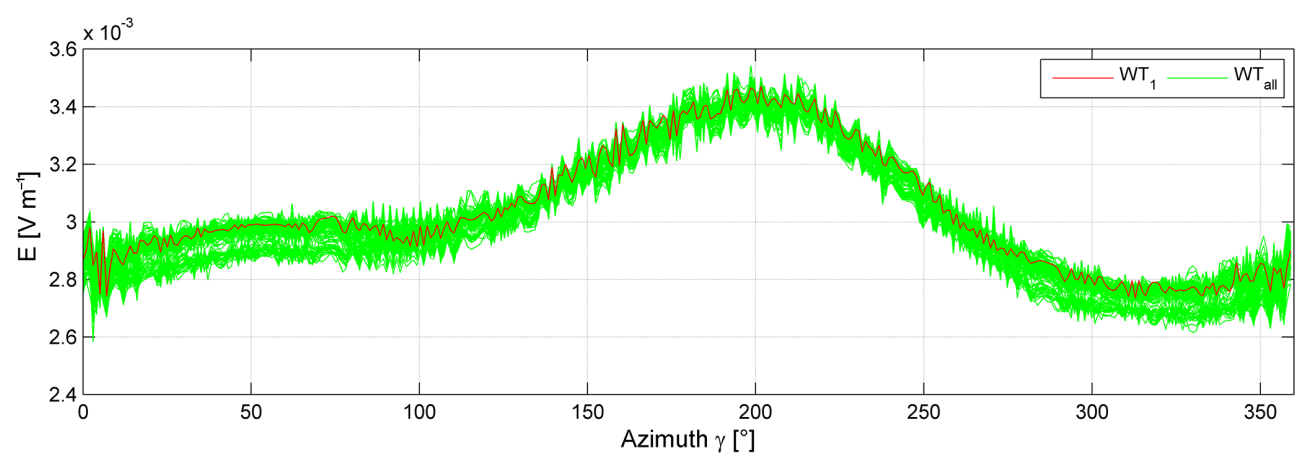

Figure 6. Electric field strength distribution along the azimuth of an orbit flight.

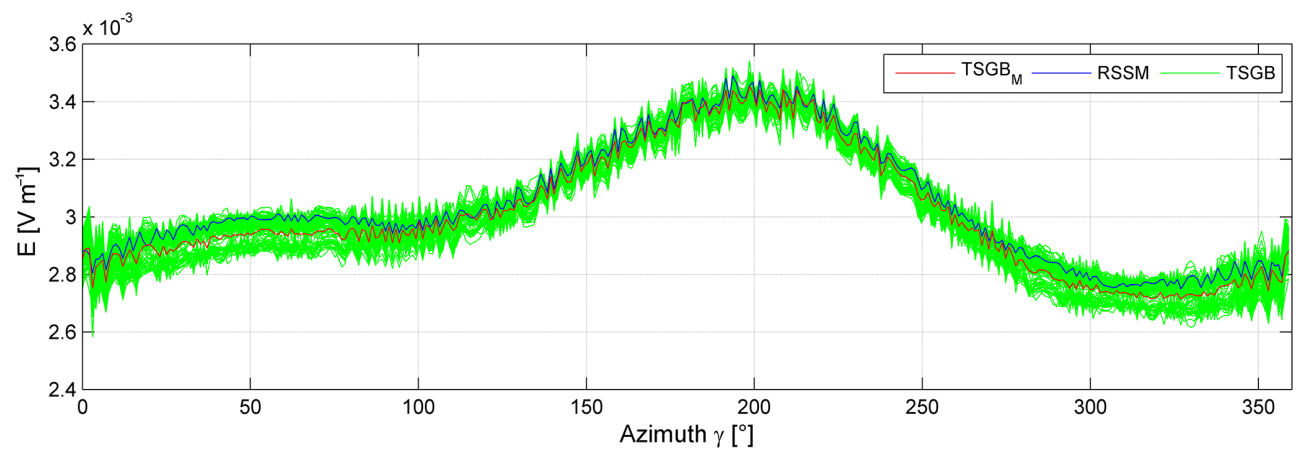

Figure 7. Electric field strength distribution according to Fig. 6 with the appropriate results for the rotationally symmetric substitution model (RSSM) compared with the arithmetic TSGB median.

\subsection{Field interval median}

Based on the assumption of the rotor blades causing both constructive and destructive interference effects on the field strength while rotating, a median field distribution is assumed that is located within the tube shaped graph bundle (TSGB) shown in Fig. 6 and representing the static tower part of WT distortion influence. To ascertain this field distribution the same simulation setup is executed with the rotationally symmetric substitution model (RSSM), shown in Fig. 2, instead of the original WT model. The appropriate results show a good compliance with the assumption as exemplary shown in Fig. 7 for the OF with $X=1 \mathrm{~km}, D=82 \mathrm{~m}, R=6 \mathrm{NM}$ and $H=6000 \mathrm{ft}$ comparing with the arithmetic TSGB median.

\subsection{Field interval width}

Considering all wind direction and rotor position constellations, the explanation related to the TSGB median in Sect. 3.2 implicates the width of the TSGB in an approximately constant range along the azimuth $\gamma$, while keeping constant the parameters $X, D, R$ and $H$. At this point the necessity to arrange the OFs coaxial with the WT as a requirement for this field distribution must be emphasized. Validating this assumption the means and standard deviations of the
TSGB interval width $E_{\mathrm{TSGB}} \mathrm{IW}$ over the azimuth $\gamma$ is calculated according to Eq. (1), as exemplary shown in Fig. 8 for all OFs with WT distance $X=1 \mathrm{~km}$. Especially for radii $R$ greater than $1 \mathrm{NM}$ this assumption is verified by the investigation. Furthermore, as expected, the TSGB width is higher in case of greater rotor diameter $D$ and is overall falling with rising radius $R$ which confirms the assumption.

$E_{\mathrm{TSGB} \_I W, \gamma}=\left|E_{\mathrm{TSGB} \_ \text {max }, \gamma}-E_{\mathrm{TSGB} \_ \text {min }, \gamma}\right|$

$\overline{E_{\text {RSSM_IW }}}=\frac{2}{360} \cdot \sum_{\gamma=1}^{360}\left|E_{\mathrm{RSSM}, \gamma}-E_{\mathrm{LFAW}, \gamma}\right|$

In order to estimate the TSGB width the averaged RSSM interval width along the azimuthal field distribution is used, since both the RSSM and the WT model are influenced by interference effects of the same manner. Determining the RSSM interval width it is not useful to base the calculation upon some filtering of the field distribution because this method disregards the difference between interference effects and intrinsic signal fractions.

Considering this aspect, the calculation of the averaged RSSM interval width $\overline{E_{\text {RSSM_IW }}}$ is based on the difference between the field distribution results of a simulation with 


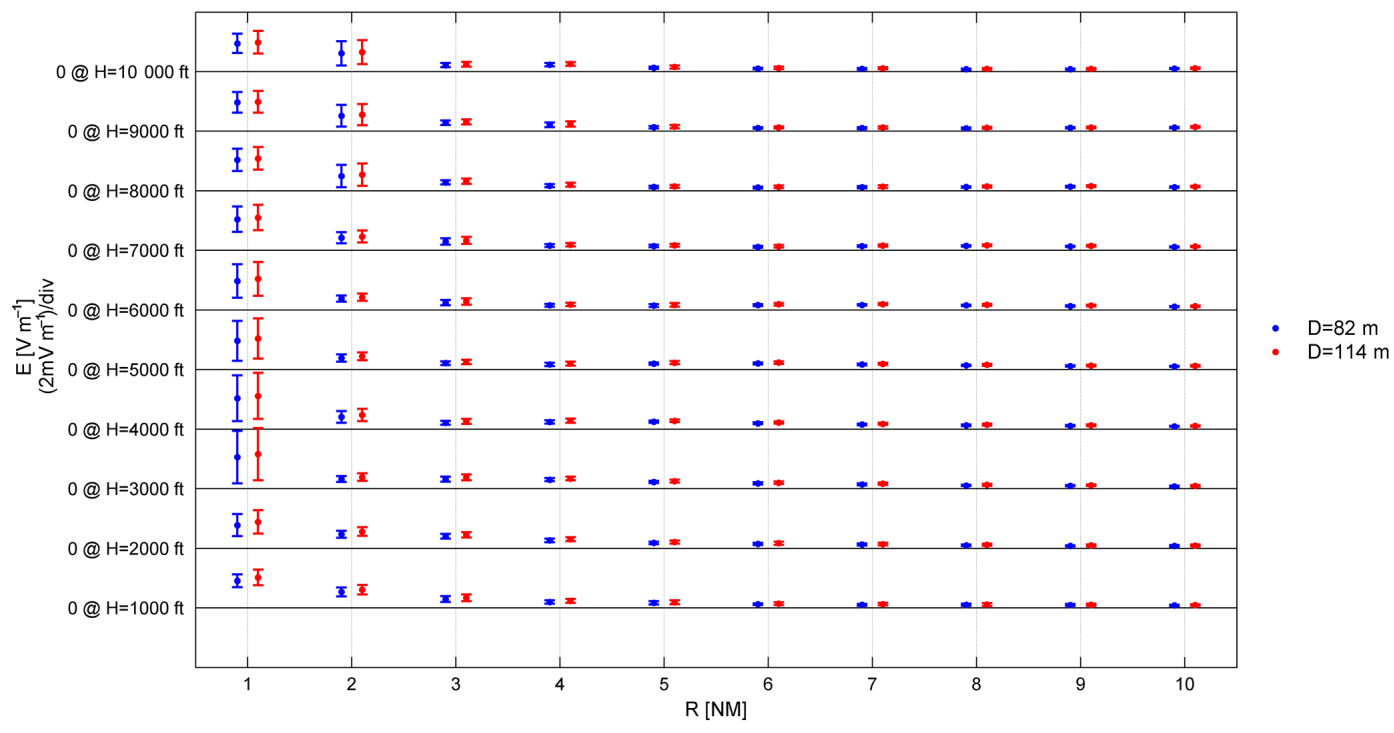

Figure 8. TSGB width means and standard deviations of all OFs with WT distance $X=1 \mathrm{~km}$.

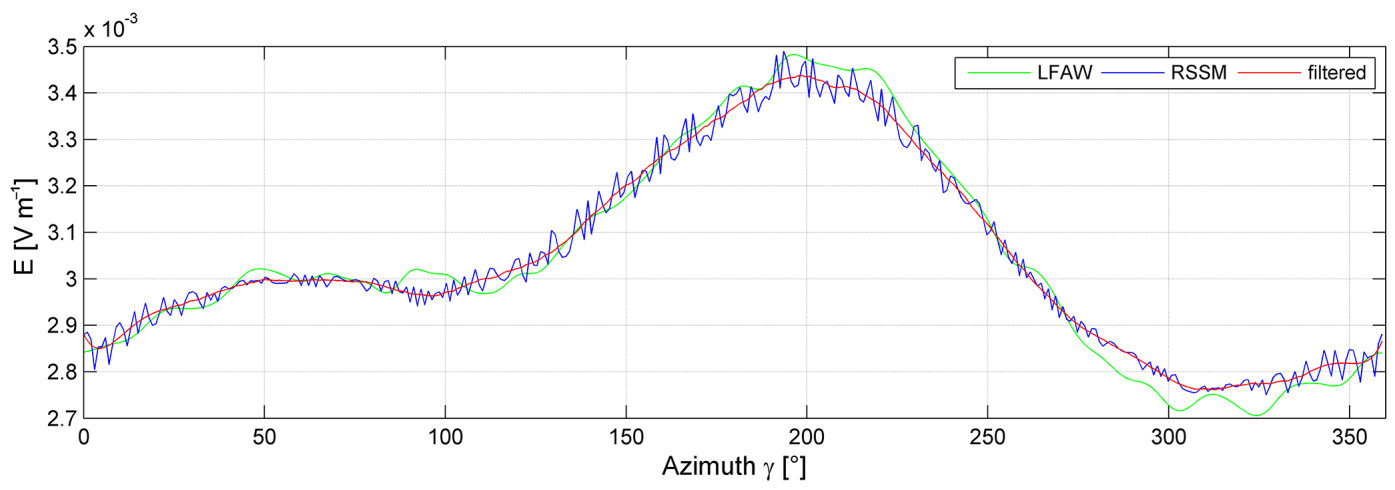

Figure 9. Field distributions for determining RSSM interval width $\overline{E_{\text {RSSM_IW }}}$ according to Eq. (2).

RSSM and linearly fitted results of the same simulation setup except for the absence of any WT model (LFAW), as shown in Fig. 9. Due to the average difference between $E_{\mathrm{RSSM}, \gamma}$ and $E_{\mathrm{LFAW}, \gamma}$ is composed of its absolute values, it has to be duplicated to determine the full averaged RSSM interval width $\overline{E_{\text {RSSM_IW }}}$, according to Eq. (2).

Regarding the ratio $\frac{E_{\mathrm{TSGB} \_ \text {IW }}}{\overline{E_{\mathrm{RSSM} \text { IW }}}}$ between the WT and RSSM interval widths, as shown in Fig. 10 for $X=1 \mathrm{~km}$, it can be stated that the mean values' range and their standard deviations are marginal. Therefor averaged proportionality factors $C_{82}=1.70$ and $C_{114}=1.96$ can be deduced by approximation for both models. As expected, the proportionality factor $C_{D}$ is higher in case of greater rotor diameter $D$.

\section{Estimation method}

As pointed out in the Sect. 1, for estimation of the electric field distribution in presence of the WT, regularities are used which are based on the results of 2080 simulation setups with $100 \mathrm{OFs}$ each. Subsequently, these regularities are calibrated by the results of RSSM and LFAW simulation. Thus, only these two simplified simulations need to be carried out for the electric field estimation.

The results of the RSSM simulation provide the estimated interval median $E_{\mathrm{EST}} \mathrm{M}$. The estimated interval width $E_{\text {EST_IW }}=\overline{E_{\text {RSSM_IW }}} \cdot C_{D}$ is constructed with both the RSSM and LFAW simulation results, according to Eq. (2), and the proportionality factor $C_{D}$ which slightly depends on the WT rotor reflexion capability affected by the blades length and shape. An exemplary field distribution estimation, created for the used OF $(X=1 \mathrm{~km}, D=82 \mathrm{~m}, R=6 \mathrm{NM}$, $H=6000 \mathrm{ft}, C_{82}=1.70$ ) with this method is shown in Fig. 11.

In order to give an overview of the estimations for the field distributions of all other OFs with the WT distance $X=1 \mathrm{~km}$ the averaged error of estimated TSGB median and width are shown using error bars in Fig. 12. 


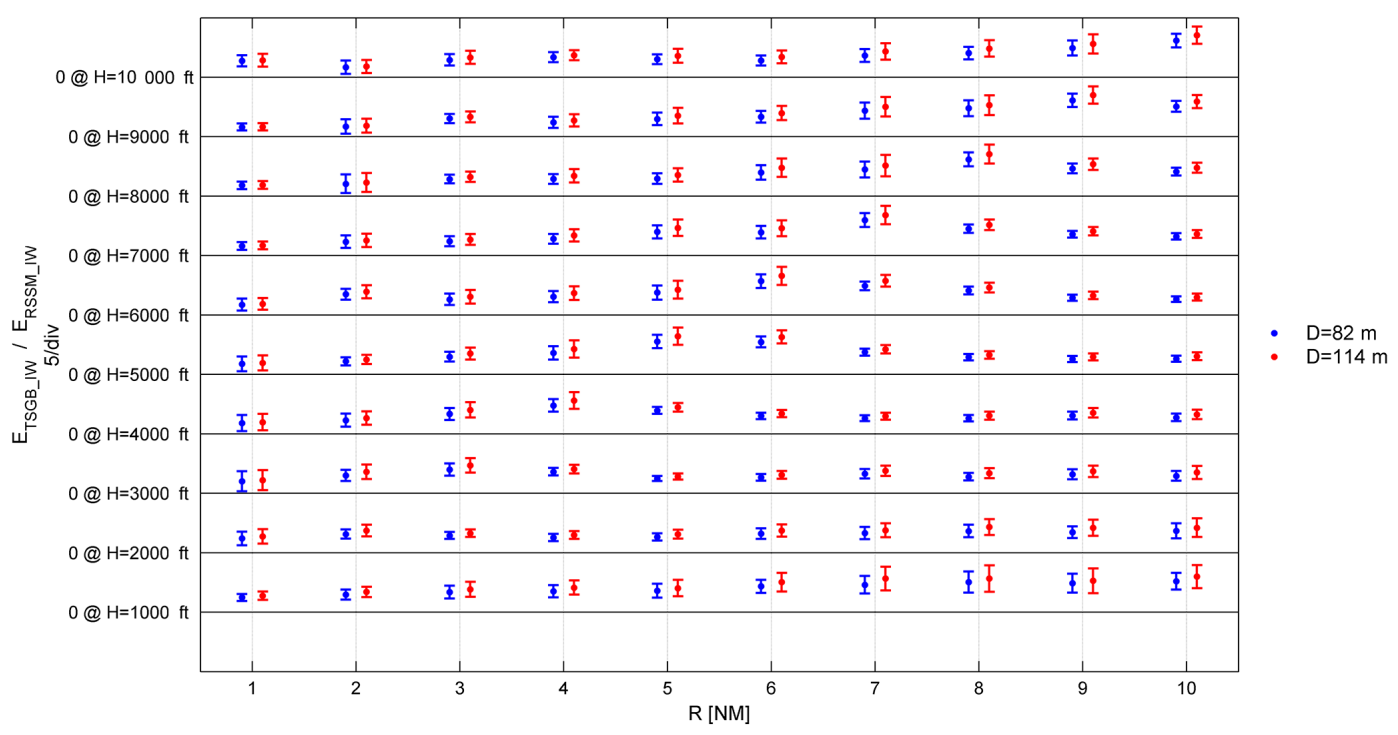

Figure 10. Ratio $\frac{E_{\mathrm{TSGB} \_\mathrm{IW}}}{\overline{E_{\mathrm{RSSM}} \mathrm{IW}}}$ between field interval widths of WT model and RSSM for $X=1 \mathrm{~km}$.

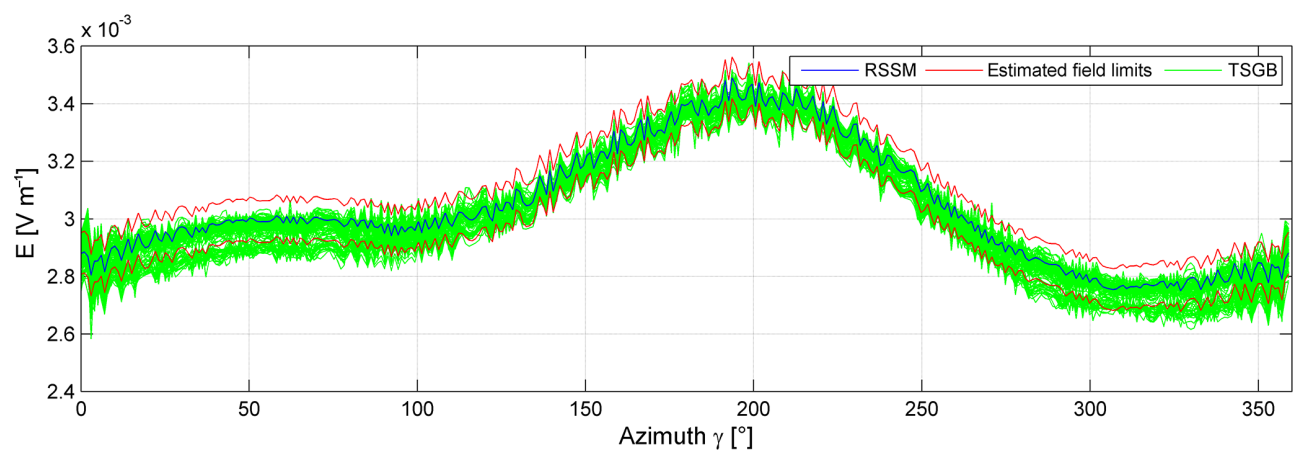

Figure 11. Field estimation for an exemplary $\mathrm{OF}$ and proportionality factor $C_{82}=1.70$.

Thereby the averaged relative TSGB median estimation error EST_M $\mathrm{M}_{\text {err }}$ is represented by the error bar median according to Eq. (3) while the averaged relative TSGB interval width estimation error EST_IW err $_{\text {is represented by the error }}$ bar width according to Eq. (4). The appropriating averaged values as a function of the WT distance are given in Fig. 13.

$$
\begin{aligned}
& \text { EST_M }_{\text {err }} \\
& =\frac{1}{360} \cdot \sum_{\gamma=1^{\circ}}^{360^{\circ}} \frac{\left|E_{\mathrm{EST} \_\mathrm{M}, \gamma, R, H}-E_{\mathrm{TSGB} \_\mathrm{M}, \gamma, R, H}\right|}{E_{\mathrm{TSGB} \_\mathrm{IW},} \gamma, R, H}
\end{aligned}
$$

$$
\begin{aligned}
& \mathrm{EST} \_I W_{\mathrm{err}} \\
& =\frac{1}{360} \cdot \sum_{\gamma=1^{\circ}}^{360^{\circ}} \frac{\left|E_{\mathrm{EST} \_\mathrm{IW}, \gamma, R, H}-E_{\mathrm{TSGB} \_\mathrm{IW}, \gamma, R, H}\right|}{E_{\mathrm{TSGB} \_\mathrm{IW}, \gamma, R, H}}
\end{aligned}
$$

The estimated relative interval width error for the OFs shown in Fig. 12 can be stated with a typical value about 1 , while the estimated relative median error is typically about $1 / 5$ of the interval width. These values can also be distinguished in Fig. 13 for $X=1 \mathrm{~km}$, as this error bar represents the averaged errors of all OFs with this $X$ value. The rising error bar values with the WT distance $X$ are constituted by the decreasing TSGB width for higher distances between DVOR and WT, caused by the free space loss. According to Eqs. (3) and (4) this leads to the rising relative error values.

\section{Conclusions}

A simulation method has been introduced to estimate the electric field strength radiated by a DVOR and distorted by a WT. The method is particularly aimed at curtailing the minimum and maximum field distortions caused by the WTs dynamic behavior like blade rotation and wind alignment without the need of simulating all possible geometric constellations. 


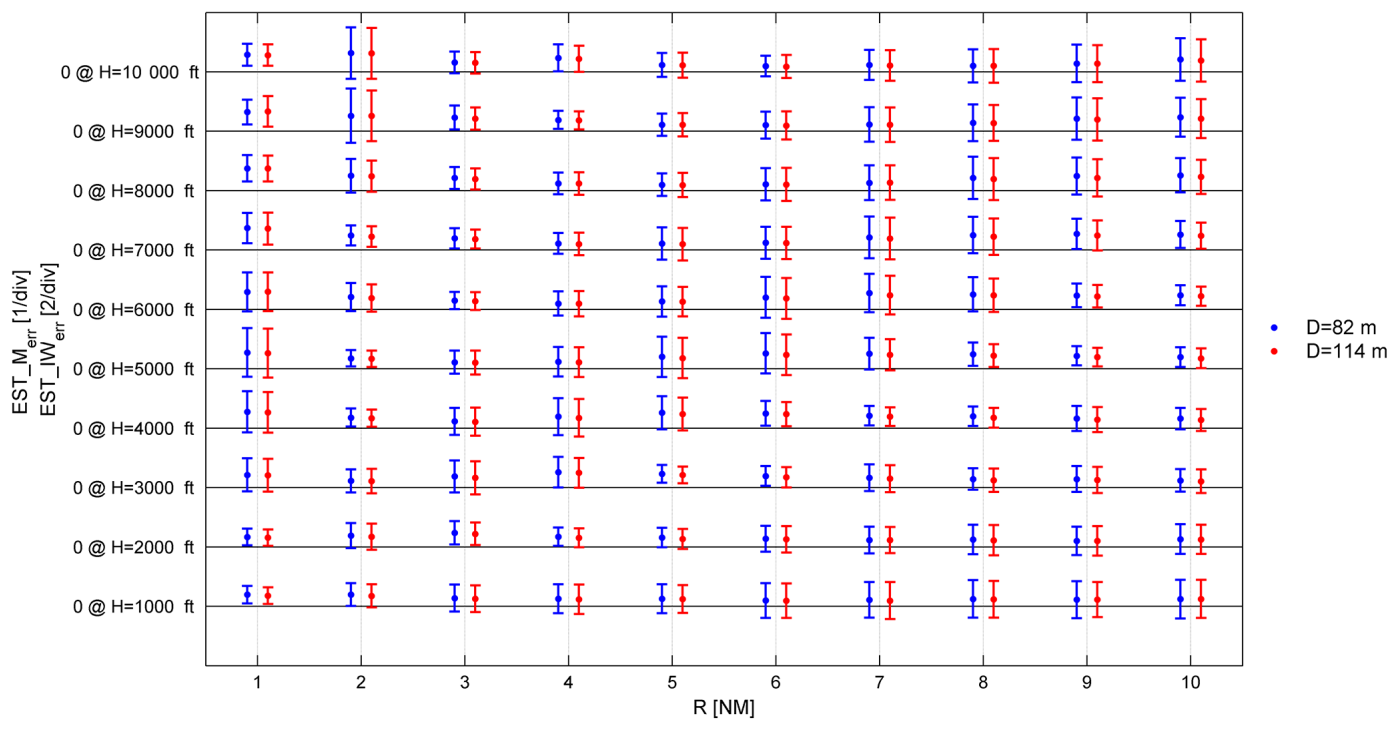

Figure 12. Field estimation error for all OFs with $X=1 \mathrm{~km}$.

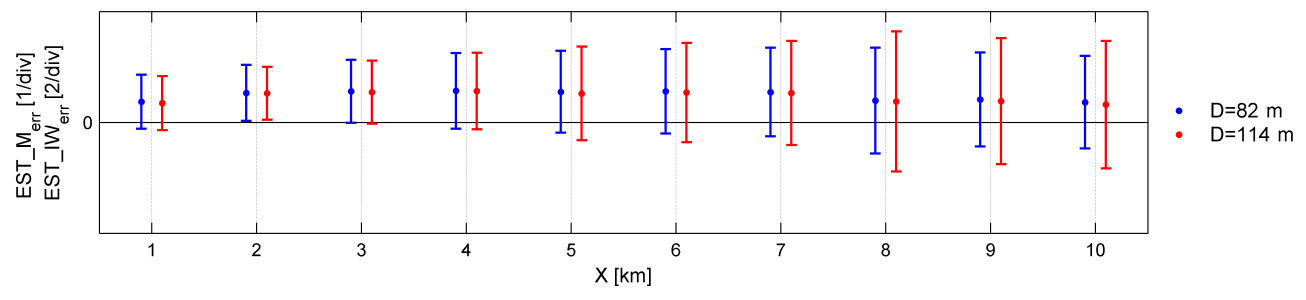

Figure 13. Field estimation error as a function of WT distance $X$.

Therefor regularities of the distortion behavior were determined by analyzing the simulation results of 2040 nonredundant WT constellations. It was worked out that the tube shaped field graph band width, created by the different WT constellations, is in a steady range provided the distance to the WT is constant. Furthermore, it is ascertained that the field graph band median can be approximated by simulating a blade-less rotationally symmetric substitution model (RSSM) while the field graph band width itself is determined based on the simulation results of the RSSM and linearly fitted results of the same simulation setup except for the absence of any WT model.

As a result this method allows to approximate worst case distortion of an omnidirectional emitted electromagnetic field in presence of a WT with the results of only two simplified simulations, which significantly reduces simulation effort. The typical overall approximation accuracy can be stated with an relative uncertainty below $1 / 4$ of the essentially field graph band width for the approximated field graph band median and an appropriate relative uncertainty factor below 1.5 for the field graph band width in regions of insignificant free space loss.
Acknowledgements. We acknowledge support by Deutsche Forschungsgemeinschaft and Open Access Publishing Fund of Leibniz Universität Hannover. Support granted by the Federal Ministry of Economy and Energy according to a resolution by the German Federal Parliament, FKZ: 0325644A-D.

The publication of this article was funded by the open-access fund of Leibniz Universität Hannover.

Edited by: T. Schrader

Reviewed by: J. Bredemeyer and one anonymous referee

\section{References}

Altair Engineering GmbH, Calwer Straße 7, 71034 Böblingen, Germany, http://www.feko.info, http://www.altair.com, 2015.

Bundesverband WindEnergie (BWE), http://www.windenergie.de/sites/default/files/attachments/page/arbeitskreisluftverkehr-und-radar/20131107-bwe-umfrage-radar.pdf (last access: 30 April 2016), 2014.

Gallardo-Hernando, B., Munoz-Ferreras, J. M., Perez-Martinez, F., and Aguado-Encabo, F.: Wind turbine clutter observations and theoretical validation for meteorological radar applications; IET Radar, Sonar a. Navigation, 5, 111-117, 2011. 
ICAO EUR DOC 015: International Civil Aviation Organization, European Guidance Material On Managing Building Restricted Areas, 2015.

Sandmann, S., Divanbeigi, S., and Garbe, H.: Reflexionseigenschaften von Windenergieanlagen im Funkfeld von Funknavigations- und Radarsystemen, Adv. Radio Sci., 13, 918, doi:10.5194/ars-13-9-2015, 2015.

Van Lil, E., Trappeniers, D., De Bleser, J., and Van de Capelle, A.: Computations of radar returns of wind turbines; Antennas and Propagation, 3rd European Conference on; Berlin, Germay, 2009. van Tonder, J. J. and Jakobus, U.: Fast multipole solution of metallic and dielectric scattering problems in FEKO; Wireless Communications and Applied Computational Electromagnetics, IEEE/ACES International Conference on, 511-514, 2005. 